\title{
A technical note on the bias in the estimation of the $b$-value and its uncertainty through the Least Squares technique
}

\author{
Laura Sandri and Warner Marzocchi \\ Istituto Nazionale di Geofisica e Vulcanologia, Sezione Bologna, Italy
}

\begin{abstract}
We investigate conceptually, analytically, and numerically the biases in the estimation of the $b$-value of the Gutenberg-Richter Law and of its uncertainty made through the least squares technique. The biases are introduced by the cumulation operation for the cumulative form of the Gutenberg-Richter Law, by the logarithmic transformation, and by the measurement errors on the magnitude. We find that the least squares technique, applied to the cumulative and binned form of the Gutenberg-Richter Law, produces strong bias in the $b$-value and its uncertainty, whose amplitudes depend on the size of the sample. Furthermore, the logarithmic transformation produces two different endemic bends in the $\log (N)$ versus $M$ curve. This means that this plot might produce fake significant departures from the Gutenberg-Richter Law. The effect of the measurement errors is negligible compared to those of cumulation operation and logarithmic transformation. The results obtained show that the least squares technique should never be used to determine the slope of the Gutenberg-Richter Law and its uncertainty.
\end{abstract}

Key words b-value - seismology - least squares technique - syntheticearthquake catalogs

\section{Introduction}

The Gutenberg-Richter Law (from now on GR Law) (Gutenberg and Richter, 1954) is certainly one of the most remarkable and ubiquitous features of seismicity worldwide. In the most common form it reads

$$
\log [N(M)]=a-b M .
$$

Mailing address: Dr. Laura Sandri, Istituto Nazionale di Geofisica e Vulcanologia, Sezione Bologna, Via Donato Creti 12, 40128 Bologna, Italy; e-mail: sandri@bo.ingv.it
In the so-called binned form of the GR Law, $N$ represents the number of events with magnitude $M$, while in the cumulative form $N$ is the number of events with magnitude larger or equal to $M$. The constants $a$ and $b$ are the coefficients of the linear relationship.

As discussed in a previous paper (Marzocchi and Sandri, 2003, from now on MS03), the scientific relevance of the GR Law is linked to its apparent ubiquity, and to the theoretical implications and meaning of its possible universality. In particular, the value of $b$, representing the opposite of the slope of the linear relationship in eq. (1.1), is considered very important. In fact, in spite of a «first order» validity of the GR Law with a constant $b$-value $\approx 1$ observed in a variety of tectonic settings, significant spatial and temporal variations in the $b$-value are found (e.g., Riedel et al., 2003; Del Pezzo et al., 2004; Legrand et al., 2004; Mandal et al., 2004; Ratchkovski et al., 2004; Wyss et al., 2004; Mur- 
ru et al., 2005; Schorlemmer et al., 2005). These possible variations are very important from a theoretical point of view, and for seismic hazard studies. We refer to our previous paper (MS03) for a deeper discussion on these issues.

As a matter of fact, a crucial aspect in inferring variations or constancy of the $b$-value is represented by the method used to determine the $b$-value and its uncertainty, given a seismic catalog, as shown in MS03. In that paper, we discussed the maximum likelihood method to estimate the $b$-value and its uncertainty, showing that incorrect formulae based on this method produce a bias in the $b$-value and an underestimation of its uncertainty. The latter implies that we might observe a fake variation in the $b$-value only because we have underestimated the uncertainty. Intentionally, in MS03 we did not consider the least squares (from now on, LS) method, because it had already been recognized (Page, 1968; Bender, 1983) that this technique applied to the problem of the estimation of the $b$-value and of its uncertainty does not have any statistical foundation. However, the LS technique is still widely employed to estimate these two quantities, both in the binned and in the cumulative form, also in recent literature (e.g., Working Group on California Earthquake Probabilities, 2003; Gruppo di Lavoro, 2004; Lopez Pineda and Rebollar, 2005).

The main purpose of this paper is to demonstrate, by means of conceptual issues, analitical formulations and numerical simulations, that the use of the LS technique in the estimation of the $b$-value and of its uncertainty leads to strongly biased estimates of these two quantities. For the correct estimation of these two quantities, we refer to the appropriate formulae given in MS03 and in references therein.

\section{Estimation of $b$ and $\sigma_{b}$ through the LS technique}

The LS estimation consists of applying linear regression analysis to the GR Law described by eq. (1.1) in the binned or cumulative form. The parameter $b$ should be the same in the two formulations, while the intercept $a$ is different in the two cases.

\subsection{Cumulative form of the GR Law}

Despite its widespread adoption in past and recent papers (Pacheco and Sykes, 1992; Pacheco et al., 1992; Karnik and Klima, 1993; Okal and Kirby, 1995; Scholz, 1997; Triep and Sykes, 1997; Main, 2000; Working Group on California Earthquake Probabilities, 2003; Gruppo di Lavoro, 2004; Lopez Pineda and Rebollar, 2005), the use of LS technique to estimate the $b$ value on the cumulative form of the GR Law does not have any statistical motivation. The strongest assumption of regression analysis, i.e., the independence of the observations, is clearly violated. In practice, the cumulative form is an integration and, therefore, it represents a filter for the high frequency noise. As a result, the uncertainty of the slope of the GR Law is certainly strongly underestimated. In order to evaluate the bias in the regression analysis applied to the cumulative form numerically, as a function of the number of earthquakes contained in the catalog, we simulated 1000 seismic catalogs, for different catalog sizes. The magnitudes $M_{i}$ are obtained by binning, with a fixed bin width of 0.1 , a continuous random variable distributed with a probability density function (pdf) given by equation

$$
f(M)=b \ln (10) 10^{-b\left(M-M_{\min }\right)}
$$

which is valid if the catalog contains earthquakes with a completeness magnitude equal to $M_{\min }$ and with a magnitude range of at least three units (see MS03). In this way, $M_{i}$ is the magnitude attached to all the synthetic seismic events with real continuous magnitude in the range $M_{i}-0.05 \leq M<M_{i}+0.05$.

Then, for each synthetic catalog, we estimated the $b$-value (from now on, using the notation $\hat{b}$ for the estimated value, and $b$ for the theoretical value) by means of the LS technique applied to the cumulative GR Law described by eq. (1.1), i.e. when $N$ is the number of events with magnitude larger or equal to $M_{i}$.

Figure 1 reports the averages of $\hat{b}$ calculated in 1000 synthetic catalogs as a function of the number of data, for the cases $b=1$ and $b=2$. The $95 \%$ confidence interval is attached to each average. There is a clear negative bias, that decreases with the number of data and ranges 
from $5 \%$ to $2 \%$. An explanation of this bias will be given in the following when we describe the effects of the logarithmic transformation in the regression analysis of the binned magnitudes.
As mentioned above, another crucial aspect concerns the estimation of the uncertainty attached to $\hat{b}$, here indicated by $\hat{\sigma}_{\hat{b}}$ and estimated as the average error on $\hat{b}$ provided by the 1000
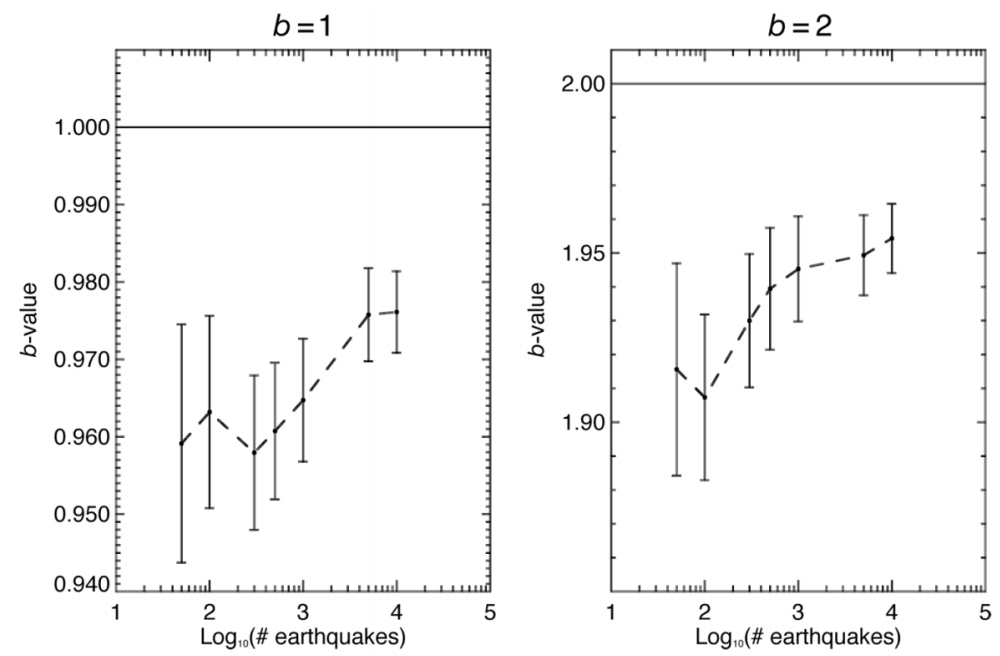

Fig. 1. Average of $\hat{b}$ (dashed line) calculated through cumulative LS technique in 1000 synthetic catalogs, as a function of the catalog size, for the cases $b=1$ (left) and $b=2$ (right). At each average is attached the 95\% confidence interval. The solid line represents the true $b$-value.
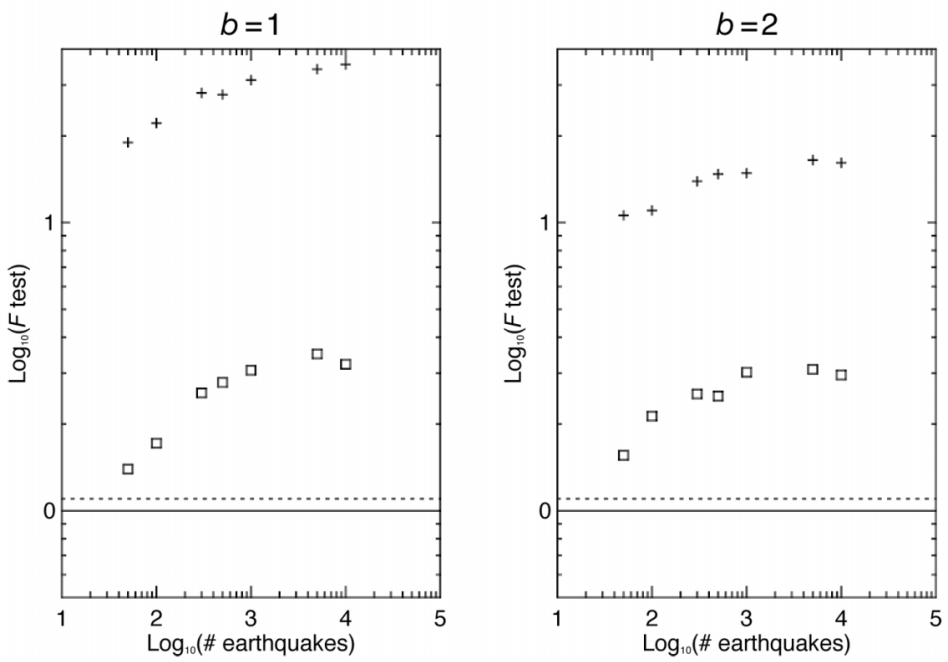

Fig. 2. $F$ test values (see eq. (2.2)) for the case of figs. 1 and 4 (see text). The plus signs represent the cumulative regression, and the squares the binned regression. The dotted line represents the critical value to reject the null hypothesis at a significance level of 0.05 . Note the logarithmic scale also on the vertical axis. 
linear regressions. In particular, we are interested in evaluating if the estimation of $\hat{\sigma}_{\hat{b}}^{2}$ is an unbiased estimator of the true variance of the $b$ estimation of $\hat{b}$ around its central value. This can be done by simulating 1000 seismic catalogs as described above. For each catalog we calculated $\hat{b}$ and $\hat{\sigma} \hat{b}$. Then, we compared the dispersion of $\hat{b}$ around its average with the average of $\hat{\sigma}_{\hat{b}}$, through the Fisher test (e.g., Kalbfleisch, 1985)

$$
F=\frac{\text { Variance of the estimation of the } b-\text { value }}{\text { Average of the square of the uncertainty }} .
$$

The null hypothesis we tested is that the average of $\hat{\sigma}_{\hat{b}}^{2}$ is equal to the variance of $\hat{b}$.

The results, reported in fig. 2, are very interesting. In particular, the Fisher test shows that $\hat{\sigma}_{\hat{b}}$ strongly underestimates the dispersion of $b$, the former being at least one order of magnitude smaller than the latter. This confirms that the uncertainty on the $b$-value estimated through the cumulative LS method is strongly underestimated.

In general, the potential factors which can bias the estimations made through the LS technique (both in the cumulative and binned form) are the logarithmic transformation of the number of events, and the presence of the measurement errors on the magnitude. By taking into account the criticism at the regression analysis applied to the cumulative form just reported, in the following we studied the impact of these factors only on the estimation of the $b$-value made by means of the regression analysis applied to the binned form.

\subsection{Effects of the logarithmic transformation}

The expected number of events for a magnitude $M$ is

$$
v_{i}=n p_{i}=n \int_{M_{i}-\Delta M / 2}^{M_{i}+\Delta M / 2} f(M) d M
$$

where $n$ is the total number of earthquakes in the catalog, $\Delta M$ is the bin width used, and $f(M)$ is given by eq. (2.1). The expected fluctuations around this value can be written as

$$
\Delta N_{i}=\sqrt{n p_{i}\left(1-p_{i}\right)} .
$$

In this frame, the observed number of events $N_{i}$ for a binned magnitude $M_{i}$ can be written as $N_{i}=$ $=v_{i}\left(1+\xi_{i}\right)$, where $\xi_{i}$ is a random variable with zero mean and standard deviation equal to $\Delta N_{i} / v_{i}$. By taking into account eqs.((2.3) and (2.4)), we can write

$$
\frac{\Delta N_{i}}{v_{i}}=\sqrt{\frac{1-p_{i}}{n p_{i}}} .
$$

It is easy to demonstrate that $\Delta N_{i} / v_{i}$ is a strictly monotonic decreasing function of $p_{i}$. This means that the expected fluctuations of the variable $\xi$ are larger for lower $p_{i}$, that is for large magnitudes.

The estimation of $b$ in the classical linear regression analysis is (see e.g., Draper and Smith, 1981)

$$
\hat{b}=-\frac{\sum M_{i} \sum\left[\log \left(N_{i}\right)\right]-n \sum M_{i}\left[\log \left(N_{i}\right)\right]}{\left(\sum M_{i}\right)^{2}-n \sum M_{i}^{2}} .
$$

If we substitute $N_{i}=v_{i}\left(1+\xi_{i}\right)$ we obtain

$$
\begin{aligned}
& \hat{b}=-\frac{\sum M_{i} \sum\left[\log \left(v_{i}\right)\right]-n \sum M_{i}\left[\log \left(v_{i}\right)\right]}{\left(\sum M_{i}\right)^{2}-n \sum M_{i}^{2}}+ \\
& -\frac{\sum M_{i} \sum\left[\log \left(1+\xi_{i}\right)\right]-n \sum M_{i}\left[\log \left(1+\xi_{i}\right)\right]}{\left(\sum M_{i}\right)^{2}-n \sum M_{i}^{2}} .
\end{aligned}
$$

If we apply the expectation operator to both sides of eq. (2.7), it is possible to verify that the bias is zero only if $E\left[\log \left(1+\xi_{i}\right)\right]=0$. This is certainly not true because $E\left[\log \left(1+\xi_{i}\right)\right]<\log$ $\left\{E\left[\left(1+\xi_{i}\right)\right]\right\}=0$.

To summarize, the application of the classical linear regression analysis to the logarithm of real data (therefore affected by errors) produces a systematic bias on the estimated slope. This is not due to the regression analysis (because the latter always provides an unbiased estimate), but it is related to its application to this type of data.

The logarithmic transformation has also another very important effect due to the discreteness of the dependent variable $N$. We have just 


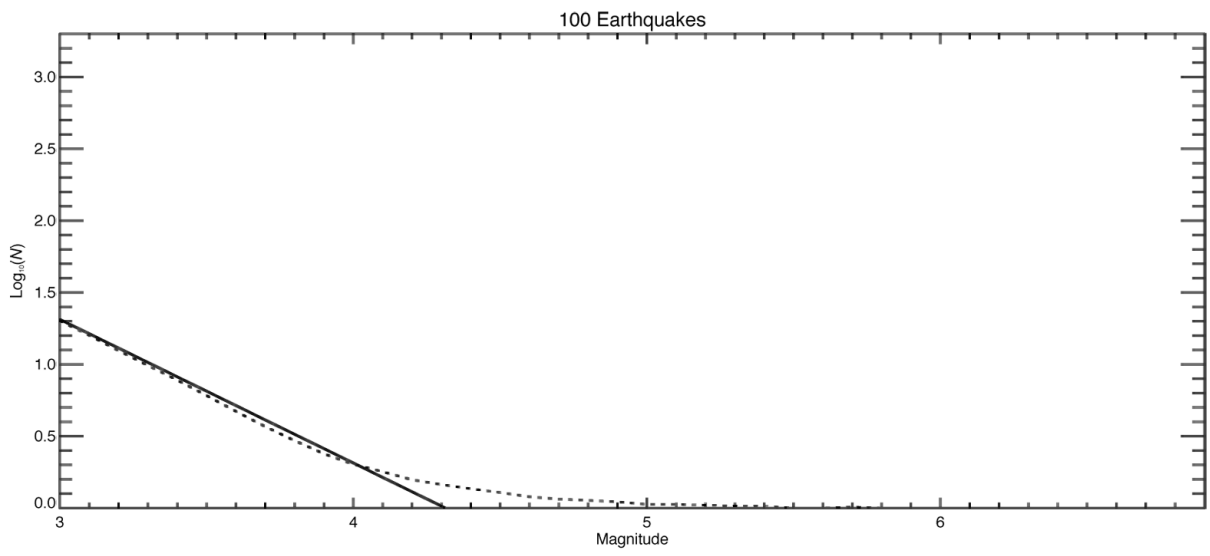

1000 Earthquakes

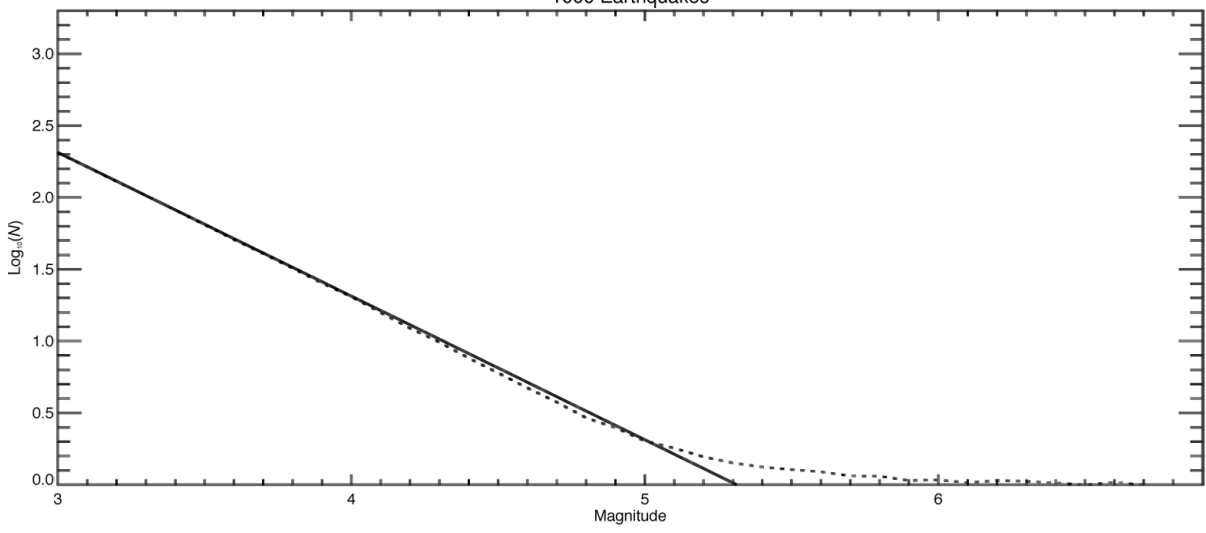

10000 Earthquakes

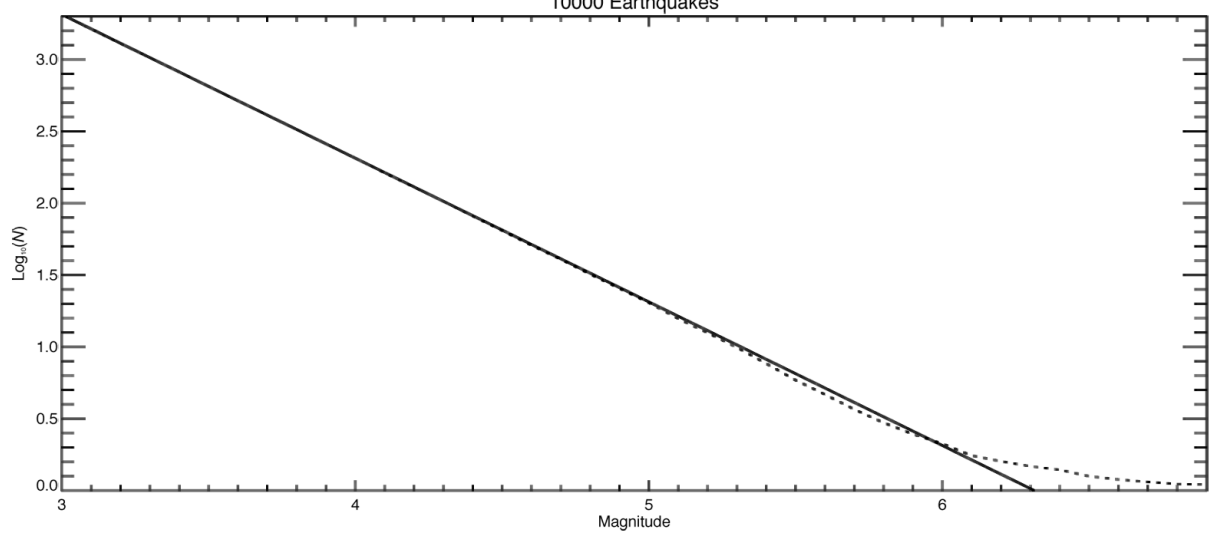

Fig. 3. Logarithm of the expected number of earthquakes $\left(\log \left(v_{i}\right)\right.$; see text) according to the GutenbergRichter Law (solid line) and average of the logarithm of the generated number of events in each of 1000 synthetic catalogs (dotted line), plotted as a function of the magnitude, for different catalog sizes (from top to bottom: 100, 1000, 10000 events per catalog). The intersection between the solid and dotted lines represents the point where the bending changes in sign. 
shown that the dispersion of the data around the curve increases with magnitude. Since we cannot compute $\log \left(N_{i}\right)$ if $N_{i}=0$, at large magnitudes, where $v$ is low (for example $v \leq 1$ ), the LS technique can take into account only the «positive» fluctuations around the mean value, i.e. the fluctuations that increase the expected number of events. This acts as a filter for the «negative» fluctuations, that is at large magnitudes (where it can be computed) $\log \left(N_{i}\right)$ tends to be overestimated compared to $\log \left(v_{i}\right)$. The global effect consists in the introduction of a systematic negative bias in the estimation of $b$, that is $\hat{b}$ is always lower than $b$. Recalling that the amplitude of the dispersion around the curve is higher for small data sets, in these cases the bias should be larger.

Figure 3 reports $\log \left(v_{i}\right)$ (see eq. (2.3)) and the averages of $\log \left(N_{i}\right)$ obtained by 1000 synthetic catalogs as a function of the magnitudes $M_{i}$. For each magnitude $M_{i}$, the average of $\log \left(N_{i}\right)$ is computed only on those catalogs for whom $N_{i} \geq 1$. At lower magnitudes, in the left part of the graph (to the left of the intersection point between the two lines in fig. 3), $\log \left(N_{i}\right)$ tends to underestimate $\log \left(v_{i}\right)$. This bias, due to the second addendum in the right side of eq. (2.7), should lead to an overestimation of the $b$ value. On the contrary, at large magnitudes (to the right of the intersection point between the two lines in fig. 3), there is a turnover in the trend of $\log \left(N_{i}\right)$ with respect to $\log \left(v_{i}\right)$. Here, $\log \left(N_{i}\right)$ tends to be larger than $\log \left(v_{i}\right)$. As mentioned above, this effect is due to the filtering of the negative fluctuations of $N_{i}$ at large magnitudes performed by the logarithmic transformation of a discrete variable. The consequence is an underestimation of the $b$-value. The results reported in fig. 3 suggest that, when the larger magnitudes are taken into account, this last bias is always predominant. As argued above, this bias is reduced by increasing the size of the data set. Instead, if the larger magnitudes are excluded (e.g., Karnik and Klima, 1993), the plot $\log \left(N_{i}\right)$ versus $M_{i}$ shows only one bend, and the $b$-value is overestimated independently on the size of the catalog.

In other words, the global effect of the logarithmic transformation of a discrete variable (the number of seismic events) introduces two bends in the GR curve. It is noteworthy that these dis- crepancies from a straight line are not linked to any physical process, but only to the mathematical transformation. Similar considerations can be made by looking at fig. 4 that reports the estimations of the $b$-value as a function of the size of the data set. The bias is very strong for small data set and it decreases as the size of the data set increases. The bias is higher in the binned case compared to the cumulative one (compare figs. 4 and 1). This is due to the fact that in the cumulative regression there are fewer zero values in the dependent variable. As shown in fig. 2, the uncertainty of the $b$-value made in the binned regression is still underestimated, even though it is better than the strong underestimations obtained by the cumulative regression.

\subsection{Effects of the measurement errors}

If we add a measurement error at the theoretical magnitude $(M)$, we obtain the «real» magnitude $(\widetilde{M})$ as a sum of two independent random variables

$$
\widetilde{M}=M+\varepsilon
$$

where $M$ is the earthquake magnitude devoid of measurement errors distributed with a pdf given by eq. (2.1), and $\varepsilon$ simulates the measurement errors distributed as a Gaussian noise. Let us consider the number of the earthquakes with a binned magnitude $M_{i}$, that is $N\left(M_{i}\right)$. By adding a Gaussian noise to each magnitude as in eq. (2.8), some events go out of and some come into the considered bin, as shown in fig. 5. The final number is $N\left(\widetilde{M}_{i}\right)$. The number of data which go out of the bin is (see fig. 5)

$$
m_{\mathrm{OL}}=m_{\mathrm{OR}}=P_{\varepsilon} N\left(M_{i}\right)
$$

where $m_{\mathrm{OL}}$ is the number of data which go into the adjacent left bin, $m_{\mathrm{OR}}$ is the number of data that go into the adjacent right bin, and $P_{\varepsilon}$ is the probability that a randomly chosen event inside the bin goes out of the bin itself when a measurement error is added. $P_{\varepsilon}$ is independent from $M_{i}$ and it depends on $\sigma_{\varepsilon}^{2}\left(P_{\varepsilon} \rightarrow 0\right.$ when $\left.\sigma_{\varepsilon}^{2} \rightarrow 0\right)$.

The number of data which come in from the left bin is (see fig. 5) 


$$
m_{\mathrm{LL}}=P_{\varepsilon} N\left(M_{i-1}\right)=P_{\varepsilon} N\left(M_{i}-\Delta M\right) .
$$

The number of data which come in from the right bin is (see fig. 5)

$$
m_{\mathrm{IR}}=P_{\varepsilon} N\left(M_{i+1}\right)=P_{\varepsilon} N\left(M_{i}+\Delta M\right)
$$

Then, we have

$$
\begin{aligned}
& N(\widetilde{M})=N(M)+P_{\varepsilon}\left[N\left(M_{i}+\Delta M\right)\right. \\
& \left.+N\left(M_{i}-\Delta M\right)-2 N(M)\right] .
\end{aligned}
$$

If eq. (1.1) holds, eq. (2.12) can be rewritten as

$$
N\left(\widetilde{M}_{i}\right)=N\left(M_{i}\right)+P_{\varepsilon} 10^{a-b M_{i}}\left(10^{-b \Delta M}+10^{b \Delta M}-2\right) .
$$

For $b \approx 1$ and $\Delta M=0.1$, as in most of the practical cases, the term of eq. (2.13) inside the round brackets is $\approx 0$. In these case $N\left(M_{i}\right) \approx N\left(\widetilde{M}_{i}\right)$, therefore the calculation of $b$ is only slightly affected by the measurement error. At the opposite, when $\Delta M$ is large and/or $b>1$, the term inside the round brackets cannot be neglected
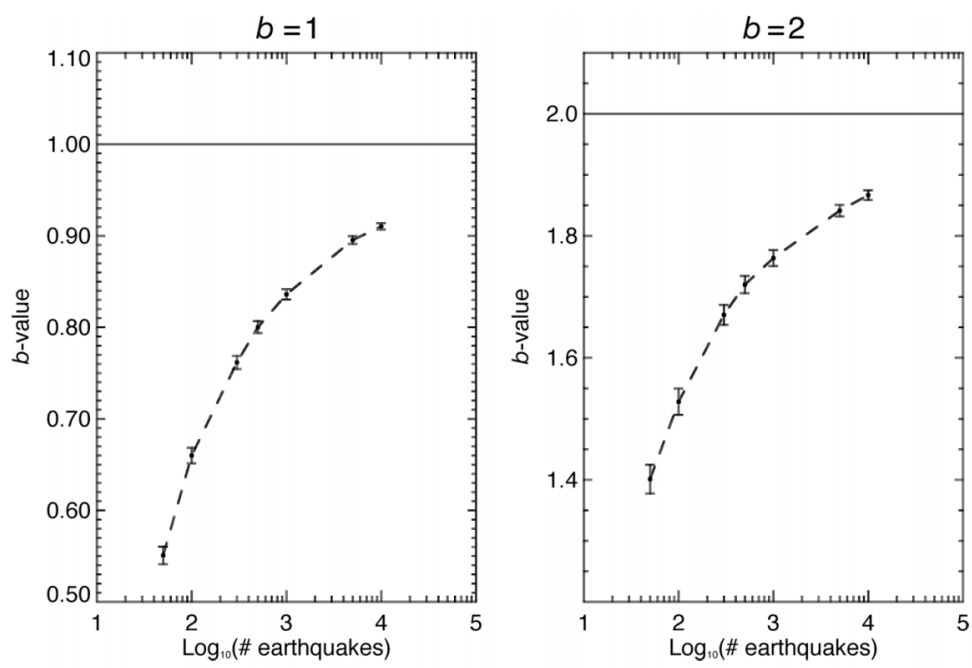

Fig. 4. Average of $\hat{b}$ (dashed line) calculated through binned LS technique in 1000 synthetic catalogs, as a function of the catalog size, for the cases $b=1$ (left) and $b=2$ (right). At each average is attached the $95 \%$ confidence interval. The solid line represents the true $b$-value.

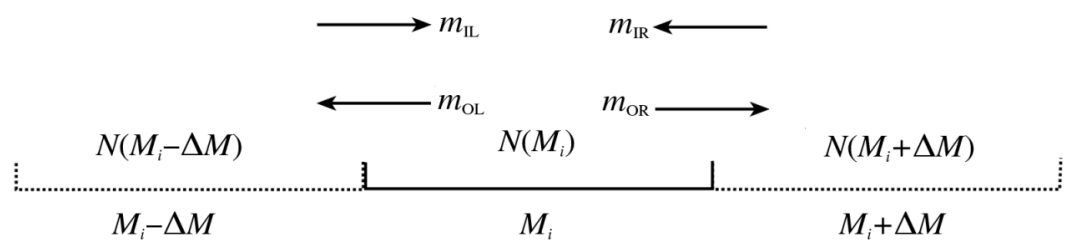

Fig. 5. Effect of the measurement errors on the number of earthquakes with binned magnitude $M_{i}$. Adding the measurement errors, the number of events that come into the bin with central magnitude $M_{i}$ from the adjacent left bin is $m_{\mathrm{IL}}$, while those coming from the adjacent right bin is $m_{\mathrm{IR}}$. The number of events that go out of the bin with central magnitude $M_{i}$ towards the adjacent left bin is $m_{\mathrm{OL}}$, while those going out towards the adjacent right bin is $m_{\mathrm{OR}}$. 

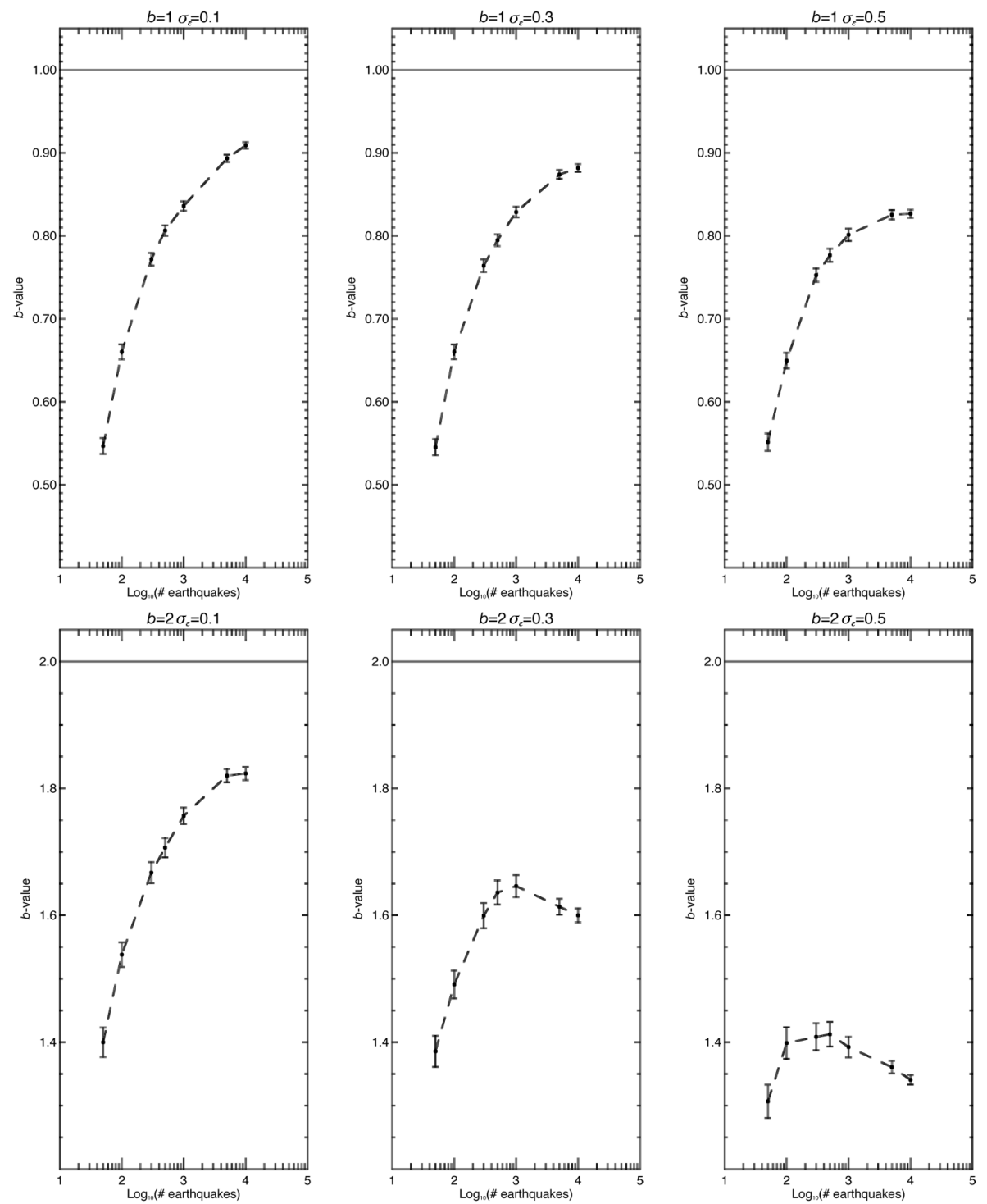

Fig. 6. Averages of $\hat{b}$ (dashed lines) calculated through binned LS technique in 1000 synthetic catalogs as a function of the catalog size, for the cases $b=1$ (top) and $b=2$ (bottom). The magnitudes have measurement errors of standard deviation $\sigma_{\varepsilon}=0.1,0.3,0.5$ (from left to right). At each average is attached the $95 \%$ confidence interval. The solid line represents the true $b$-value.

and $N\left(M_{i}\right) \neq N\left(\widetilde{M}_{i}\right)$. This obviously introduces a further bias in the $b$-value estimation.

Figure 6 reports the effect of the added measurement errors. As argued before, the largest difference with the case without measurement errors reported in fig. 4 is for $b=2$, where the term of eq. (2.13) inside the round brackets is significantly different from zero. 
Since $P_{\varepsilon}$ depends on the amplitude of the measurement error $\left(\sigma_{\varepsilon}\right)$, eq. (2.13) explains also the proportionality of the bias with $\sigma_{\varepsilon}$ noted in fig. 6. As regards the estimation of the uncertainty of the $b$-value, the results reported in fig. 7 show that the addition of the measurement errors does not produce any further bias.
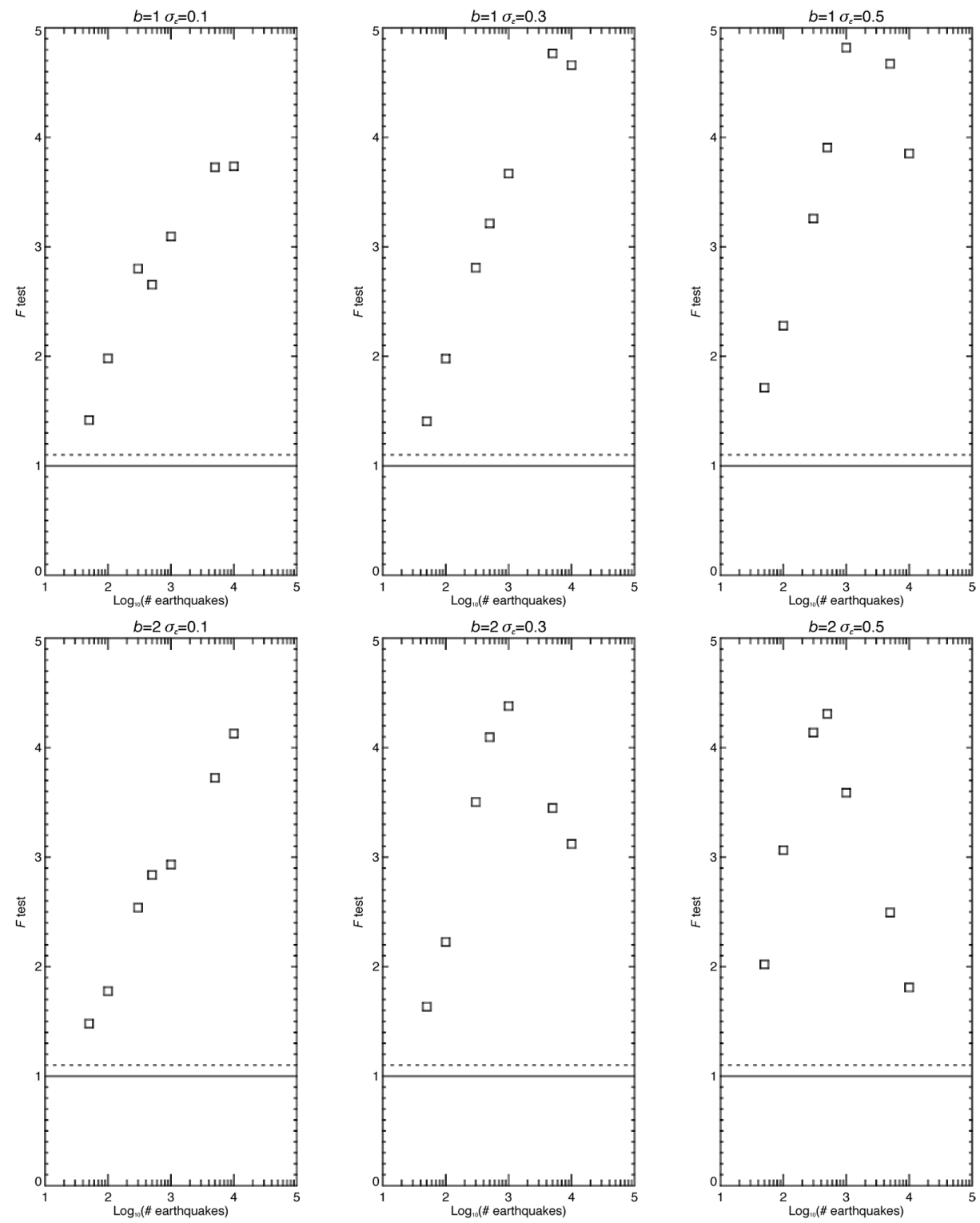

Fig. 7. $F$ test values (see eq. (2.2)) for the case of fig. 6 (see text). The dotted line represents the critical value to reject the null hypothesis at a significance level of 0.05 . 


\section{Final remarks}

We have studied conceptually, analytically, and numerically the biases introduced by some factors (the cumulation operation, the logarithmic transformation, and the measurement errors on the magnitudes) on the estimation of the $b$-value and of its uncertainty by means of the least squares technique. We have found many problems.

First, besides violating a basic assumption of the regression analysis, the use of the cumulative form of the Gutenberg-Richter Law leads to a very strong underestimation of the uncertainty of the $b$-value, due to the filtering effect of the cumulation operation.

Secondly, the logarithmic transformation of the discrete random number of events produces a significant bias in the estimation of the $b$-value both for the cumulative and binned form of the Gutenberg-Richter Law. Moreover, the bias strongly depends on the size of the data set; this means that two samples of different sizes coming from the same statistical distribution will have a significant difference in the $b$-value estimated. The same logarithmic transformation produces two endemic bends of different signs in the $\log (N)$ versus $M$ plot. These bends are not linked to the physical process. This means that some departures from a straight line in the plot $\log (N)$ versus $M$ do not invalidate the Gutenberg-Richter Law.

Finally, the influence of the measurement errors appears to be less important than the bias introduced by the logarithmic transformation.

The main purpose of this paper was to show that the estimation of the $b$-value by means of the least squares method is strongly biased and its uncertainty results heavily underestimated. In this view, this work intended to make clear why this method should not be used to determine the slope of the Gutenberg-Richter Law, and to make inferences about its constancy or variations. In practice, any spatial or temporal variation of the $b$-value obtained by this method has to be regarded with a strong skepticism. On the other hand, unbiased estimates of the $b$-value and of its uncertainty can be obtained by using the correct formulae, based on the maximum likelihood method, that have been analitically and numerically tested in Marzocchi and Sandri (2003) (see also references therein).

\section{REFERENCES}

BENDER, B. (1983): Maximum likelihood estimation of $b$ values for magnitude grouped data, Bull. Seismol. Soc. Am., 73, 831-851.

Del Pezzo, E., F. Bianco, S. Petrosino and G. Saccorotti (2004): Changes in the coda decay rate and shear-wave splitting parameters associated with seismic swarms at Mt. Vesuvius, Italy, Bull. Seismol. Soc. Am., 94 (2), 439452.

DraPer, N.R. and H. SMITH (1981): Applied Regression Analysis (John Wiley \& Sons Ltd., New York), 2nd edition, pp. 305.

Group on California EARThQuaKe Probabilities (2003): Earthquake probabilities in the San Francisco Bay region: 2002-2031, U.S. Geol. Surv. Open File Rep. 03-214.

GRUPPO DI LAVORO (2004): Redazione della mappa di pericolositá sismica prevista dall'Ordinanza PCM 3274 del 20 marzo 2003, Rapporto Conclusivo per il Dipartimento della Protezione Civile (INGV, Milano-Roma, aprile 2004), pp. 65.

GutenberG, B. and C. Richter (1954): Seismicity of the Earth and Associated Phenomena (Princeton University Press, Princeton, New Jersey), 2nd edition, pp. 310.

KalbFleisch, J.D. (1985): Probability and Statistical Inference (Springer-Verlag, New York), 2nd edition, pp. 364.

KARNIK, V. and K. KlimA (1993): Magnitude-frequency distribution in the European-Mediterranean earthquake regions, Tectonophysics, 220, 309-323.

Legrand, D., D. Villagomez, H. YePes and A. CAlahorraNO (2004): Multifractal dimension and $b$-value analysis of the 1998-1999 Quito swarm related to Guagua Pichincha Volcano activity, Ecuador J. Geophys. Res., 109 (B1), B01307, doi: 10.1029/2003JB002572.

Lopez PinedA, L. and C.J. Rebollar (2005): Source characteristics of the $M_{w} 6.2$ Loreto earthquake of 12 March 2003 that occurred in a transform fault in the middle of the Gulf of California, Mexico, Bull. Seismol. Soc. Am., 95 (2), 419-430.

MaIN, I. (2000): Apparent breaks in scaling in the earthquake cumulative frequency-magnitude distribution: fact or artifact?, Bull. Seismol. Soc. Am., 90, 86-97.

MANDAL, P., B.K. RASTOGi, H.V.S. SATYANARAYANA and M. KousAlya (2004): Results from local earthquake velocity tomography; implications toward the source process involved in generating the 2001 earthquake in the lower crust beneath Kachchh (India), Bull. Seismol. Soc. Am., 94 (2), 633-649.

MARzocchi, W. and L. SANDri (2003): A review and new insights on the estimation of the $b$-value and its uncertainty, Ann. Geophysics, 46 (6), 1271-1282.

Murru, M., C. Montuori, R. Console and A. Lisi (2005): Mapping of the $b$-value anomalies beneath Mt. Etna, Italy, during JulyAugust 2001 lateral eruption, Geophys. Res. Lett., 32 (5), L05309, doi: 10.1029/2004GL021545.

OKAL, E.A. and S.H. KIRBY (1995): Frequency-moment distribution of deep earthquake; implications for the seismogenic zone at the bottom of Slabs, Phys. Earth Planet. Inter., 92, 169-187.

PACHECO, J.F. and L.R. Sykes (1992): Seismic moment catalog of large shallow earthquakes, 1900 to 1989 , Bull. Seismol. Soc. Am., 82, 1306-1349.

Pacheco, J.F., C.H. Scholz and L.R. Sykes (1992): 
Changes in frequencysize relationship from small to large earthquakes, Nature, 355, 71-73.

PAGE, R. (1968): Aftershocks and microaftershocks of the Great Alaska earthquake of 1964, Bull. Seismol. Soc. Am., 58, 1131-1168.

RATChKovski, N.A., S. WiEmer and R.A. HANSEN (2004): Seismotectonics of the Central Denali Fault, Alaska, and the 2002 Denali Fault earthquake sequence, Bull. Seismol. Soc. Am., 94 (6), Part B, 156-174.

Riedel, C., T. Petersen, F. Theilen and S. Neben (2003): High $b$-values in the leaky segment of the Tjornes fracture zone north of Iceland; are they evidence for shallow magmatic heat sources?, J. Volcanol. Geotherm. Res., 128 (1-3), 15-29.

Scholz, C.H. (1997): Size distributions for large and small earthquakes, Bull. Seismol. Soc. Am., 87, 1074-1077.
SCHORLEMmer, D., S. WIEMER and M. Wyss (2005): Variations in earthquake-size distribution across different stress regimes, Nature, 437, doi: 10.1038/nature04094.

TRIEP, E.G. and L.R. SyKES (1997): Frequency of occurrence of moderate to great earthquakes in intracontinetal regions: implications for changes in stress, earthquake prediction, and hazard assessment, J. Geophys. Res., 102, 9923-9948.

Wyss, M., C.G. SAMmis, R.M. NAUdeAu and S. WIEMER (2004): Fractal dimension and $b$-value on creeping and locked patches of the San Andreas Fault near Parkfield, California, Bull. Seismol. Soc. Am., 94 (2), 410-421.

(received December 27, 2006; accepted March 21, 2007) 